1 Universidade Estadual do Oeste do Paraná

(Unioeste) - Cascavel (PR), Brasil.

brunazack@hotmail.com

\section{Acidente de trabalho grave: perfil epidemiológico em um município do oeste do Paraná}

\author{
Major accident at work: epidemiological profile in a western Paraná \\ municipality
}

Bruna Tais Zack', Claudia Ross', Leda Aparecida Vanelli Nabuco de Gouvêa', Nelsi Salete Tonini $\mathbf{1}$

DOI: $10.1590 / 0103-1104202012707$

RESUMO Objetivou-se conhecer o perfil epidemiológico dos acidentes de trabalho graves em município do oeste do Paraná no período 2014-2018. Tratou-se de pesquisa quantitativa, retrospectiva, descritiva realizada por meio do Sistema de Informação de Agravos de Notificação (Sinan). Realizou-se análise descritiva e estatística. Totalizaram-se 1.741 notificações, predominando a faixa etária de 25 a 45 anos, sexo masculino, raça branca, ensino médio completo, residência na cidade estudada e área urbana. A principal ocupação foi a de empregado doméstico nos serviços gerais, prevalecendo acidente típico, nas instalações do contratante, empresas não terceirizadas, carteira assinada, Comunicação de Acidente de Trabalho não preenchida, e tempo de serviço inferior a um ano. Acontecidos predominantemente no horário comercial, sem outros trabalhadores envolvidos. A maioria recebeu atendimento médico notificado na unidade hospitalar, mão mais atingida, regime de tratamento hospitalar mais frequente até evolução para incapacidade temporária. Destacou-se o grupo de quedas e traumas. Totalizaram-se 39 óbitos com principal ocupação de pedreiro. A maioria das variáveis foram significativas para ocorrência dos acidentes e desfecho. A ocorrência de óbito foi relacionada à parte do corpo atingida e à classificação da lesão. O conhecimento do perfil epidemiológico pode subsidiar estratégias de saúde na área de saúde do trabalhador.

PALAVRAS-CHAVE Acidentes de trabalho. Notificação de acidentes de trabalho. Epidemiologia.

\begin{abstract}
The objective of this study was to know the epidemiological profile of individuals notified for serious work accidents in a municipality in western Paraná, in the period 2014-2018. This was a quantitative, retrospective, descriptive research carried out through the Information System for Notifiable Diseases (Sinan). Descriptive and statistical analysis was performed. There were a total of 1,741 notifications, with a predominance of the age group from 25 to 45 years old, male, white race, complete high school, residency in the studied city and urban area. The main occupation: domestic servant in general services, with a typical accident prevailing, at the contractor's premises, non-outsourced companies, a formal contract, Work Accident Report not completed, and length of service less than one year. Happened predominantly during business hours, with no other workers involved. Most received medical care, notified at the hospital, the hand most affected, the most frequent hospital treatment regime, the evolution to temporary disability. The group of falls and traumas stood out. There were 39 deaths with the main occupation of masons. Most variables were significant for the occurrence of accidents and outcome. The occurrence of death was related to the body part affected and the classification of the injury. Knowledge of the epidemiological profile can support health strategies in the area of occupational health.
\end{abstract}

KEYWORDS Accidents, occupational. Occupational accidents registry. Epidemiology. 


\section{Introdução}

A Vigilância em Saúde do Trabalhador (Visat) tem por objetivo a proteção e promoção à saúde dos trabalhadores por meio de atenção integral. A principal estratégia do Ministério da Saúde é a Rede Nacional de Atenção Integral à Saúde do Trabalhador (Renast), composta pelos Centros Estaduais e Regionais de Referência em Saúde do Trabalhador (Cerest), os quais visam a ações para melhoria das condições de trabalho e qualidade de vida dos trabalhadores'.

No ano de 2004, é oficializada a primeira portaria que regulamenta a notificação dos agravos relacionados à saúde de trabalhador ${ }^{2}$. Desde então, ocorreram melhorias em sua vigilância. A Política Nacional de Saúde do Trabalhador tem por finalidade definir os princípios, as diretrizes e as estratégias a serem observadas e implantadas no Sistema Único de Saúde (SUS), aliando-se a um conjunto de políticas públicas em função da necessidade de vigilância do agravo ${ }^{3}$. A portaria vigente sobre notificação de doenças e agravos de saúde elenca o acidente de trabalho grave como notificação compulsória 4 .

Segundo a Lei $n^{\circ} 8.213$ de 24 de julho de 1991, são considerados acidentes de trabalho aqueles decorrentes do exercício laboral ${ }^{5}$. O acidente de trabalho grave é aquele cuja causa possa ser relacionada à atividade laboral e que resulte em mutilação e repercussão funcional incapacitante ou fatal ${ }^{6}$. É de comunicação obrigatória imediata à autoridade de saúde via notificação ao Sistema de Informação de Agravos de Notificação (Sinan) ${ }^{\mathbf{4}}$.

A definição de caso de acidente de trabalho grave, de acordo com o Sinan, é aquele relacionado à atividade laboral, acontecido na execução da atividade ou no percurso da casa para o trabalho, resultando em morte, mutilação ou em menores de 18 anos 7.

Estima-se que ocorram em todo mundo aproximadamente 317 milhões de acidentes de trabalho por ano, sendo que 2,34 milhões de pessoas morrem anualmente em função de acidentes por sua atividade laboral ${ }^{8}$.
Nesse contexto, destaca-se no cenário paranaense $o$ acidente de trabalho grave como o segundo agravo mais notificado, perdendo apenas para o acidente de trabalho com exposição à material biológico 9 .

Diante do exposto, este estudo tem como pergunta norteadora: Qual o perfil epidemiológico dos acidentes de trabalho graves em município do oeste do Paraná no período de 2014-2018?

\section{Material e métodos}

Trata-se de pesquisa quantitativa, retrospectiva, descritiva que tem por objetivo identificar o perfil de trabalhadores notificados por acidente de trabalho grave ao Sinan no município de Cascavel (PR) no período de 01 de janeiro de 2014 a 31 dezembro de 2018.

A pesquisa foi desenvolvida na divisão de Visat da Secretaria Municipal de Saúde do município de Cascavel (PR), que é localizada na região oeste do Estado e possui 328.454 habitantes. $\mathrm{O}$ estudo contemplou a análise das notificações por acidente de trabalho grave ao Sinan no município para o período. O período proposto para o estudo foi determinado conforme disponibilidade e confiabilidade dos dados registrados no Sinan. O instrumento de coleta de dados foi construído com base em informações relevantes da ficha de notificação de acidente de trabalho grave, sendo aplicado ao TabWin ${ }^{\circledR}$ para obtenção e importação dos dados do Sinan?.

A análise estatística foi dividida em dois momentos. Primeiramente os dados foram tabulados em planilhas do programa Microsoft Excel ${ }^{\circledR}$, submetidos à estatística descritiva para verificar a significância ( $\mathrm{p}$-valor $<0,05$ ). Posteriormente, realizada análise de agrupamento para identificar similaridade entre os indivíduos nas variáveis e descobrir quais delas tinham relação com a ocorrência e com os óbitos por acidente de trabalho grave. Para isso, utilizou-se a distância de Manhattan para o cálculo da matriz de similaridades, sendo que 
o número de grupos não foi fixado. A partir dos grupos obtidos, analisou-se quais deles continham um número muito baixo de sujeitos (inferior a 40), os quais foram descartados do conjunto de dados por serem considerados casos isolados.

Na sequência, com o novo conjunto de dados, realizou-se a caracterização geral utilizando os grupos restantes e o teste de Quiquadrado para independência. Em situações de ferimento do pressuposto de frequência mínima esperada igual a 5 , foi utilizado como teste de associação o método de Monte Carlo, com $5 \%$ de significância.

$\mathrm{Na}$ etapa de verificação do risco de óbito dos trabalhadores, apenas as variáveis que se mostraram associadas estatisticamente aos agrupamentos foram utilizadas ( $\mathrm{p}$-valor $<0,05$ ). Para tal, foi ajustado um modelo matemático pelo método de regressão logística binária. As variáveis significativas nos Qui-quadrados para independência foram consideradas como explicativas, e o desfecho do atendimento aos pacientes, com óbito ou não, como variável resposta do modelo. $\mathrm{O}$ ajuste do modelo de regressão foi verificado pela estatística de Hosmer\&Lemeshow.

Construiu-se uma curva ROC (Receiver Operating Characteristic) para o modelo de regressão com a finalidade de avaliar sua habilidade em representar a realidade. Nessa curva, foram representados os valores de sensibilidade e especificidade do modelo ajustado. Todas as análises foram realizadas no programa licenciado XLSTat ${ }^{\circledR}$ versão 2017.

A pesquisa foi aprovada pelo Comitê de Ética em Pesquisa com Seres Humanos (CEP) da Universidade Estadual do Oeste do Paraná sob o parecer $n^{\circ} 3.423 .313$ e CAAE: 15675419.3.0000.0107.

\section{Resultados}

Em um primeiro momento, foi realizada análise descritiva dos resultados encontrados e identificada 1741 notificações de acidente de trabalho grave no município de Cascavel (PR) para o período estudado, conforme se observa na tabela 1.

Tabela 1. Distribuição de frequência absoluta e relativa de indivíduos notificados para acidente de trabalho grave conforme ano de ocorrência. Cascavel (PR).

\begin{tabular}{lrrrrrrr}
\hline Ano & $\mathbf{2 0 1 4}$ & $\mathbf{2 0 1 5}$ & $\mathbf{2 0 1 6}$ & $\mathbf{2 0 1 7}$ & $\mathbf{2 0 1 8}$ & Total & p-valor \\
\hline Casos (\%) & $362(21 \%)$ & $441(25 \%)$ & $245(14 \%)$ & $280(16 \%)$ & $413(24 \%)$ & $1741(100 \%)$ & $<0,05$ \\
\hline
\end{tabular}

Fonte: Elaboração própria.

O período do ano em que ocorreu a maior concentração de acidente de trabalho grave foi no segundo semestre entre as semanas epidemiológicas 28 a $52(\mathrm{n}=967 / 55,6 \%)$, para todos os anos estudados.
A maior frequência de acidente de trabalho grave ocorreu em indivíduos com 25 a 45 anos, do sexo masculino, raça branca, com ensino fundamental incompleto e em residentes da zona urbana, como descrito na tabela 2.

Tabela 2. Distribuição de frequência absoluta e relativa para a caracterização sociodemográfica de indivíduos notificados para acidente de trabalho grave. Cascavel (PR)

\begin{tabular}{lcr}
\hline Idade & Total \% & p-valor \\
\hline 14 a 18 & $207(11,9 \%)$ & $<0,0001$ \\
19 a 24 & $272(15,7 \%)$ & \\
25 a 45 & $816(46,8 \%)$ & \\
\hline
\end{tabular}




\begin{tabular}{|c|c|c|}
\hline 46 a 50 & $153(8,8 \%)$ & \\
\hline 51 a 60 & $211(12 \%)$ & \\
\hline Mais de 60 & $82(4,8 \%)$ & \\
\hline Sexo & Total \% & $\mathrm{p}$-valor \\
\hline Feminino & $251(14,4 \%)$ & $<0,0001$ \\
\hline Masculino & $1489(85,6 \%)$ & \\
\hline Não consta & $1(0,05 \%)$ & \\
\hline Raça/cor & Total \% & p-valor \\
\hline Branca & $1354(77,6 \%)$ & $<0,0001$ \\
\hline Preta & $50(3 \%)$ & \\
\hline Amarela & $7(0,4 \%)$ & \\
\hline Parda & $311(18 \%)$ & \\
\hline Ignorado & $17(1 \%)$ & \\
\hline Indígena & $1(0,05 \%)$ & \\
\hline Escolaridade & Total \% & $\mathrm{p}$-valor \\
\hline Analfabeto & $12(0,7 \%)$ & $<0,0001$ \\
\hline Ensino fundamental incompleto & $520(30 \%)$ & \\
\hline Ensino fundamental completo & $438(25 \%)$ & \\
\hline Ensino médio completo & $456(26 \%)$ & \\
\hline Ensino superior completo & $43(2,5 \%)$ & \\
\hline Ignorado & $241(14 \%)$ & \\
\hline Não se aplica & $10(0,6 \%)$ & \\
\hline Não consta & $21(1,2 \%)$ & \\
\hline Zona de residência & Total \% & $\mathrm{p}$-valor \\
\hline Urbana & $1592(91,4 \%)$ & $<0,0001$ \\
\hline Rural & $116(6,7 \%)$ & \\
\hline Periurbana & $3(0,2 \%)$ & \\
\hline Ignorado & $2(0,1 \%)$ & \\
\hline Não consta & $28(1,6 \%)$ & \\
\hline
\end{tabular}

Fonte: Elaboração própria.

O município de residência relatado pela maioria dos indivíduos notificados foi a cidade onde realizou-se o estudo ( $\mathrm{n}=1358 / 78 \%)$. As três ocupações pertencentes ao mesmo grupo estatístico e que apresentaram maior destaque foram a de empregado doméstico nos serviços gerais ( $n=197 / 11,3 \%)$, pedreiro $(n=167 / 9,6 \%)$ e alimentador de linha de produção ( $n=125 / 7,2 \%$ ).

Quanto ao tipo de acidente, o mais comum foi o típico ( $n=1522 / 87,4 \%)$, sendo que a maioria dos acidentes ocorreu nas instalações do contratante $(n=1072 / 61,6 \%)$. A empresa, na maioria dos casos, não era terceirizada (n=Z996/57,2\%). Quanto à situação trabalhista, a maioria dos indivíduos apresentou carteira assinada ( $n=1047 / 60,1 \%)$, ou seja, trabalhadores cuja contratação era regida pela Consolidação das Leis do Trabalho (CLT).

Não ocorreu, na maioria dos casos, a realização da Comunicação de Acidente de Trabalho (CAT) ( $n=931 / 53,5 \%)$. Ocorreram mais notificações de trabalhadores com tempo de serviço inferior a um ano ( $n=616 / 35,4 \%)$.

O horário em que se sucedeu a maioria dos acidentes de trabalho graves foi no período das 
7h00 às 17h00s (n=1353/77,8\%), não havendo, na maioria dos casos, outros trabalhadores envolvidos ( $n=1583 / 90,9 \%)$.

A maioria dos trabalhadores notificados para acidente de trabalho grave recebeu atendimento médico ( $\mathrm{n}=1688 / 97 \%)$. A unidade de atendimento com a mais significativa realização de notificações foi o Hospital Universitário do Oeste do Paraná (HUOP), localizado na cidade do estudo, Cascavel (PR), que é referência regional para atendimento em saúde em nível intermediário e alta complexidade ( $\mathrm{n}=548 / 31,5 \%)$, seguida de duas Unidades de Pronto Atendimento (UPA) do mesmo município ( $\mathrm{n}=429 / 24,6 \%$ e $\mathrm{n}=308 / 17,7 \%$, respectivamente).

Tratando-se das partes do corpo atingidas no acidente, a mão foi a mais atingida $(\mathrm{n}=600 / 34,5 \%)$, seguida de membro superior ( $\mathrm{n}=360 / 20,7 \%$ ) e membro inferior ( $n=225 / 13 \%)$. No segundo e terceiro campos da ficha de notificação referente a outras partes do corpo atingidas, a maioria foi preenchida como 'não se aplica' (n=1452/83,4\% e $\mathrm{n}=1656 / 95,1 \%$, respectivamente), revelando que, na maioria dos acidentes notificados, apenas uma parte do corpo foi atingida.

$O$ regime de tratamento que mais se destacou foi o hospitalar (n=966/55,5\%), seguido do ambulatorial ( $\mathrm{n}=722 / 41,5 \%$ ) e da evolução dos casos mais frequentes para incapacidade temporária ( $\mathrm{n}=1287 / 73,9 \%)$. No mecanismo do acidente, segundo Classificação Estatística Internacional de Doenças e Problemas Relacionados com a Saúde, décima edição (CID-10) ${ }^{\mathbf{1 0}}$, o mais frequente foi o grupo de quedas ( $\mathrm{n}=1312 / 75,3 \%$ ) e a lesão mais frequente foi o grupo dos traumas ( $\mathrm{n}=1575 / 90,7 \%)$.

Quanto à mortalidade em função do agravo, ocorreram 39 óbitos (2,2\%) diretamente relacionados ao acidente de trabalho grave, dentre os casos estudados. Dentre esses óbitos, as principais ocupações ou funções exercidas pelos acidentados foram pedreiro $(n=7 / 18 \%)$, seguido de empregado doméstico nos serviços gerais $(n=4 / 10,3 \%)$ e de motorista de caminhão ( $n=4 / 10,3 \%)$.

Em um segundo momento da análise dos dados, foi realizada a análise por agrupamento para verificar a similaridade entre os sujeitos a fim de se verificar quais variáveis apresentam relação quando se trata de notificações por acidente de trabalho grave e quais estão relacionadas à ocorrência de óbito nesses casos. Nessa, evidenciou-se a existência de nove grupos. Desses nove grupos, com base no número de elementos (número de indivíduos superior a 40), foram mantidos quatro grupos (G1- $\mathrm{n}=1332$; G2- n=41; G3- n=305; G8- n=48). Ao todo, 15 indivíduos pertencentes aos outros grupos foram excluídos por não serem similares aos demais, por serem muito diferentes em relação aos outros casos notificados, podendo ser situações incomuns de acidentes que estatisticamente pode afetar as conclusões finais desta análise.

Na caracterização geral com base nos agrupamentos por grupos (G1, G2, G3 e G8; n= 1726), pode-se perceber que todas as variáveis quantitativas mostraram associação estatística com os grupos (p-valor<0,05) (tabela 3$)$.

Tabela 3. Médias \pm desvios-padrão (DP) das variáveis quantitativas vinculadas ao acidente de trabalho grave, associadas aos agrupamentos, em relação aos agrupamentos. Cascavel (PR)

\begin{tabular}{lccr}
\hline Variáveis & Grupos & Média DP & P \\
\hline Tempo de trabalho (em anos) & 1 & $8,3 \pm 9,9$ & 0,002 \\
& 2 & $8,8 \pm 10,2$ & \\
& 3 & $4,8 \pm 8,1$ & \\
& 8 & $8,4 \pm 9,0$ & \\
\hline Hora do acidente & 1 & $12,5 \pm 4,3$ & $<0,001$ \\
& 2 & $13,2 \pm 4,0$ & \\
\hline
\end{tabular}


Tabela 3. (cont.)

\begin{tabular}{|c|c|c|c|}
\hline Variáveis & Grupos & Média DP & $\mathbf{P}$ \\
\hline & 3 & $12,7 \pm 5,2$ & \\
\hline & 8 & $13,0 \pm 4,3$ & \\
\hline \multirow[t]{4}{*}{ Hora após o início da jornada } & 1 & $5,5 \pm 6,3$ & $<0,001$ \\
\hline & 2 & $8,2 \pm 9,2$ & \\
\hline & 3 & $7,4 \pm 7,2$ & \\
\hline & 8 & $4,2 \pm 3,8$ & \\
\hline \multirow[t]{4}{*}{ Semana epidemiológica } & 1 & $27,7 \pm 14,8$ & $<0,001$ \\
\hline & 2 & $25,1 \pm 15,2$ & \\
\hline & 3 & $30,5 \pm 13,9$ & \\
\hline & 8 & $32,2 \pm 11,7$ & \\
\hline
\end{tabular}

Fonte: Elaboração própria.

Ainda, na caracterização geral com base nos agrupamentos por grupos, em relação às variáveis qualitativas - ano, idade, sexo, gestante, raça, escolaridade, zona, situação trabalhista, local onde ocorreu o acidente, se o empregador era empresa terceirizada, CID do acidente, tipo de acidente, se houve outros trabalhadores atingidos, ocorreu atendimento médico, partes do corpo atingidas, CID da lesão, regime de tratamento, evolução do caso, óbito, preenchimento da CAT -, observou-se que apenas as variáveis 'sexo', 'gestante', se 'houve outros trabalhadores atingidos' e se 'ocorreu atendimento médico' não apresentaram associação estatística com nenhum dos agrupamentos formados, ou seja, não foram significativas na formação de tais grupos.

As variáveis quantitativas e qualitativas estatisticamente significativas ( $\mathrm{p}$-valor $<0,05$ ) foram relacionadas com o desfecho do indivíduo que sofreu o acidente de trabalho grave, se óbito ou não, por meio do modelo de regressão logística binária. Ao todo, 17 variáveis quantitativas e qualitativas compuseram o modelo, sendo elas: tempo de trabalho em anos, hora do acidente, hora após o início da jornada, semana epidemiológica, raça, escolaridade, zona, local onde ocorreu o acidente, situação trabalhista, ser o empregador é uma empresa terceirizada, tipo de acidente, partes do corpo atingidas, CID do acidente, CID da lesão, regime de tratamento, evolução do caso, e ocorrência de CAT.
Devido ao elevado número de variáveis utilizadas na regressão, são exibidas na tabela 4 apenas as variáveis e respectivas categorias que foram estatisticamente significativas no modelo de regressão, sendo elas: partes do corpo atingidas (membro superior), evolução do caso (óbito), ocorrência de CAT (marcado como ignorado), e CID da lesão no grupo de (traumatismos envolvendo múltiplas lesões). O modelo apresentou um ajuste adequado segundo a estatística de Hosmer\&Lemeshow (Qui-quadrado= 4,34, $\mathrm{GL}=8, \mathrm{p}=0,8252$ ).

O modelo gerado demonstrou que indivíduos que tiveram o membro superior atingido no acidente de trabalho apresentaram 6,471 vezes mais risco de irem a óbito do que aqueles que não tiveram essa parte do corpo atingida (OR=6,471; IC95\%: 1,089 - 38,436). Os sujeitos que apresentaram a CID da lesão na categoria de traumatismos envolvendo múltiplas lesões do corpo exibiram 11,666 vezes mais chances de irem a óbito do que aqueles que não tiveram esta lesão (OR=11,666; IC95\%: 3,078 - 44,209). Aqueles cuja CAT foi marcada como ignorada exibiram baixa probabilidade de óbito (OR=0,052; IC95\%: 0,005 - 0,528). Em relação à evolução do caso, os trabalhadores cujo desfecho foi óbito por acidente de trabalho grave apresentaram uma relação direta com a variável resposta do modelo (óbito), com o valor odds ratio elevado (tabela 4). 
Tabela 4. Parâmetros obtidos para o modelo criado por meio da aplicação de regressão logística com o intuito de encontrar os fatores associados ao óbito de vítimas de acidente de trabalho grave. Cascavel (PR)

\begin{tabular}{llrrr}
\hline Fontes & & Valores & p-valor & OR [IC95\%] \\
\hline Intercepto & $-6,608$ & $<0,001$ & \\
Partes do corpo atingidas & Membro superior & 1,867 & 0,039 & $6,471[1,089-38,436]$ \\
Evolução do caso & Óbito por acidente de trabalho grave & 5,717 & $<0,001$ & $304,267[30,144-3071,196]$ \\
Fez CAT? & Ignorado & $-2,939$ & 0,012 & $0,052[0,005-0,528]$ \\
CID da lesão & Traumatismos envolvendo múlti- & 2,456 & $<0,001$ & $11,666[3,078-44,209]$ \\
& plas lesões do corpo & & & \\
\hline
\end{tabular}

Fonte: Elaboração própria.

A partir do modelo de regressão, as probabilidades foram estimadas, ordenadas e plotadas em um gráfico, fornecendo a curva ROC (gráfico 1). A área sob a curva ROC mostrou que o modelo de probabilidades estimadas conseguiu prever aproximadamente $96,5 \%$ das variáveis associados ao desfecho.

Gráfico 1. Curva ROC para o modelo de regressão logística binária com fatores preditivos ao desfecho de vítimas de acidentes de trabalho graves.

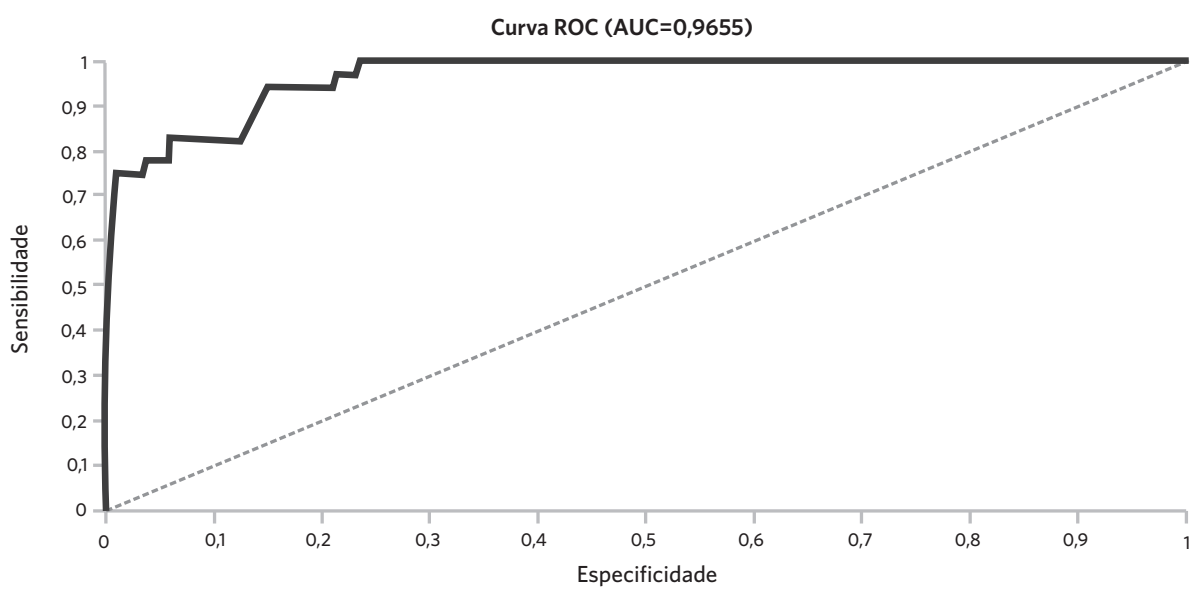

Fonte: Elaboração própria.

\section{Discussão}

No mundo, milhões de pessoas morrem em decorrência de acidentes e doenças relacionadas ao trabalho"1. Um estudo do ano de 2018 revelou que, no Brasil, o número de acidentes de trabalho, bem como a mortalidade decorrente, é elevado, chegando a 717.911 acidentes no ano 2013, com 2.797 óbitos. A região sul do país lidera o no quesito acidentes de trabalho, com 158.113 casos e 1.879 óbitos em 2013, sendo a fonte de tais estudos o Sinan ${ }^{\mathbf{1 2}}$.

Segundo os dados recentes do Observatório de Segurança e Saúde no Trabalho (OIT), no Brasil, entre o período de 2000 e 2018, houve 623,8 mil CAT informadas no sistema de 
informações específico das CAT (Siscat), dos quais 20 mil evoluídos a óbito. No município de Cascavel (PR), houve, no mesmo período 2,4 mil notificações, das quais nove evoluídas a óbito ${ }^{13}$.

Destaca-se que, no Brasil, o acidente de trabalho é considerado um agravo de notificação compulsória à autoridade de saúde contemplando $o$ acidente grave, fatal, em crianças e adolescentes e com exposição à material biológico 4 .

Neste estudo, analisaram-se os acidentes de trabalho graves ocorridos no município de Cascavel (PR) notificados ao Sinan.

$\mathrm{Na}$ análise descritiva, verificou-se que os anos de 2014, 2015 e 2018 apresentaram o maior número de notificações e que, para todos os anos estudados, a maior frequência ocorreu no segundo semestre.

O perfil sócio demográfico dos trabalhadores acidentados mostrou a predominância do sexo masculino e da faixa etária de 25 a 45 anos, corroborando outros estudos sobre a temática ${ }^{14-16}$. Em estudo realizado em Pernambuco, os achados também mostraram a predominância do sexo masculino e faixa etária de 30 a 44 anos $^{16}$. Em se tratando especificamente de acidente de trabalho grave, estudo realizado em São José do Rio Preto observou a predominância do sexo masculino e faixa etária de 25 a 45 anos ${ }^{17}$.

O último boletim epidemiológico paranaense sobre saúde do trabalhador (20062016) mostra que o sexo masculino e a faixa etária de 20 a 34 anos apresentaram o maior número de acidentes de trabalho ${ }^{9}$, sendo tal característica também verificada em nível nacional, de acordo com dados do Ministério da Previdência Social ${ }^{\mathbf{8}, \mathbf{9}}$. Neste estudo, ainda se destaca que a faixa etária de 14 a 18 anos teve 207 (11,9\%) casos notificados, e a de mais de 60 anos de idade, 82 (4,8\%) notificações. Mesmo não sendo estatisticamente significativo, tal achado merece atenção e possíveis estudos futuros acerca das causas de acidente de trabalho grave nessas faixas etárias, sobretudo entre adolescentes.

A raça de cor branca, predominante neste estudo, assemelha-se ao perfil da população exposta a acidente de trabalho no Estado, de acordo com os registros do caderno estatístico paranaense ${ }^{9}$. No entanto, quando comparada a outras regiões do país observam-se divergências: estudo realizado no estado da Bahia verificou que $62,9 \%$ dos acidentados notificados eram pardos e $13,4 \%$ negros $^{18}$.

Ressalta-se, nesse contexto, que cada região brasileira tem suas especificidades e características etno-raciais e que para cada uma delas pode-se observar a predominância ou não de determinada raça: na região sul há maior concentração da raça branca e na Bahia, da raça negra ${ }^{19}$.

A escolaridade predominante foi o nível fundamental incompleto ( $\mathrm{n}=520 / 30 \%$ ), convergindo com aquela encontrada para a população brasileira em pesquisas nacionais ${ }^{\mathbf{1 9}, 20}$, sendo também mais frequente a ocorrência de acidentes nesse grupo ${ }^{15,19,21}$.

É possível inferir uma associação de tal achado às características das atividades laborais mais observadas neste estudo: empregado doméstico nos serviços gerais, pedreiro e alimentador de linha de produção, ocupações que geralmente não exigem elevado nível de escolaridade ${ }^{15,19}$.

O trabalhador de serviços gerais, segundo a Classificação Brasileira de Ocupações, é aquele que desenvolve atividade no domicílio do empregador, como administração do lar, faxina, cuidado com plantas ou animais domésticos ${ }^{22}$.

Um estudo que descreveu o perfil dos acidentados em um município de Curitibanos, estado de Santa Catarina, também obteve resultados semelhantes, sendo a ocupação mais associada o alimentador de linha de produção ${ }^{23}$. No Paraná, recentemente, a ocupação com maior frequência de acidentes de trabalho grave foi a de 'pedreiro'9 e no país, a de 'trabalhadores de serviços' $(17,9 \%)^{8}$.

Ainda, nesse sentido, segundo o Instituto Paranaense de Desenvolvimento Econômico e Social (Ipardes) ${ }^{\mathbf{2 4}}$, no ano de 2019, no estado do Paraná, foram ofertadas 74.075 vagas de emprego formal, das quais 41.821 para o setor de 'serviços', 10.082 para a construção civil, 9.199 para o ramo industrial e as restantes 
foram destinadas ao comércio e agricultura. As ocupações mais observadas no estudo coincidem com aquelas mais ofertadas no Estado.

Em outro trabalho que corrobora em parte os achados deste estudo, verificou-se que os ramos de atividade mais frequentemente relacionados a acidentes de trabalho foram serviços, comércio, indústria e construção civil, respectivamente21. Estudo mais recente mostrou que os setores com mais acidentes de trabalho no Brasil são as indústrias, agropecuárias e serviços ${ }^{12}$.

O município e zona de residência da maioria dos indivíduos notificados foram o município de Cascavel (PR) e zona urbana, demonstrando que residem, trabalham e são atendidos no próprio município do acidente. A maioria dos casos recebeu atendimento médico, sendo o HUOP a unidade com mais notificações, dado que o trauma é seu perfil de atendimento, o que converge com o regime de tratamento mais ocorrido, o hospitalar.

As unidades hospitalares concentram significativa parte dos atendimentos dos acidentes de trabalho ${ }^{25}$, sendo os atendimentos e internações hospitalares responsáveis por elevados custos relacionados aos acidentes de trabalho. Estima-se que, incluindo as internações, os gastos das doenças e acidentes de trabalho chegam a $\mathrm{R} \$ 71$ bilhões por ano no Brasil ${ }^{15}$, sendo sua ocorrência considerada um problema de saúde pública por, dentre outros motivos, sua repercussão econômica ${ }^{26}$.

Neste estudo, o tipo de acidente foi significativamente o típico. Eles são considerados como aqueles decorrentes da característica da atividade laboral desenvolvida pelo indivíduo ${ }^{8}$ e também são os mais prevalentes em outras pesquisas semelhantes sobre o tema ${ }^{19,27}$.

Durante 0 ano de 2017, foram registrados na Previdência Social cerca de 549,4 mil acidentes do trabalho, sendo predominante o acidente típico (75,5\%), o sexo masculino (68,9\%), a faixa etária de 25 a 34 anos (32,6\%). Os achados deste estudo, em se tratando desses aspectos, assemelham-se ao panorama nacional ${ }^{\mathbf{8}}$.

A maior ocorrência das notificações foi de acidente de trabalho grave acontecidos nas instalações do contratante, em que a empresa, na maioria dos casos, não era terceirizada e o trabalhador tinha a carteira assinada. No País, o trabalho informal vinha seguindo uma tendência decrescente desde 2012, embora, no ano de 2017, tal situação tenha se modificado em decorrência de mudanças no panorama político e econômico do Brasil.

Dados recentes revelam que a maior parte da população brasileira economicamente ativa do setor privado trabalha no estabelecimento do contratante (74,4 milhões de pessoas) ${ }^{\mathbf{2 8}}$, compatível com o achado deste estudo.

Segundo Pialarissi26, a informalidade contratual trabalhista afeta os interesses dos trabalhadores, sendo, então, vantajosa para o trabalhador a formalização em função da regulamentação dos direitos trabalhistas e sociais, garantindo, caso necessário, amparo ao mesmo. O pesquisador associa ainda a terceirização à intensificação das jornadas e a baixos salários.

Acerca do registro em carteira de trabalho, observa-se em outros estudos realizados na Bahia ${ }^{\mathbf{1 8}}$ e São José do Rio Preto ${ }^{\mathbf{1 7}}$ resultados semelhantes, revelando que a maioria dos casos de acidentes de trabalho ocorreu com empregados registrados. A informalidade e a precariedade dos sistemas de informação podem tender a não demonstrar os reais acidentes de trabalho quando se tratam daqueles acontecidos com profissionais sem regulamentação ${ }^{26}$.

A não realização ou preenchimento da CAT foi predominante neste estudo, achado semelhante a outro estudo sobre o tema ${ }^{25}$. Segundo Fontana e Grillo ${ }^{23}$, quando acontece um acidente de trabalho, a empresa tem obrigação legal do seu preenchimento, referente aos profissionais celetistas, a fim de registrar o agravo mediante possibilidade da necessidade de auxílio governamental. Nesse mesmo estudo, houve uma perda amostral de 2,6\% em função do preenchimento precário das CAT analisadas, demonstrando que, além da não adesão, há também o preenchimento inadequado. 
O preenchimento da CAT, mesmo sendo uma obrigação legal, no ano de 2007, passou a não ser mais requisito obrigatório para concessão de auxílio acidente ou doença, causando diminuição na adesão e seu preenchimento em todo território nacional, de acordo com a Previdência Social ${ }^{8}$.

Quanto ao tempo de execução da atividade laboral, foi mais frequente a notificação do acidente em trabalhadores que desempenhavam a função há menos de um ano. Estudo realizado em um centro referência de saúde do trabalhador em Minas Gerais sugere que a inexperiência não está totalmente relacionada à ocorrência de acidente de trabalho, informando que pode ser decorrente do mau manuseio de ferramentas comuns ao dia-a-dia, sendo a falta do uso de Equipamentos de Proteção Individual (EPIs) (51,1\%) e a falta de treinamento (69,5\%) mais relevantes ${ }^{25}$.

Nesse item, a prevenção de acidente de trabalho e a capacitação do trabalhador sobre a importância da segurança na execução de sua atividade laboral podem ser fortes aliadas ${ }^{29}$. Dentre as várias medidas de prevenção, destaca-se o uso dos EPIs na manutenção da segurança do trabalhador. Outro estudo revela que, quanto à causalidade, a maioria dos acidentes de trabalho poderiam ter sido evitados com o uso adequado dos EPIs ${ }^{12}$.

O uso de EPIs e de Equipamentos de Proteção Coletiva (EPCs) visam à minimização de riscos para acidentes, sendo direito dos trabalhadores o seu recebimento quando admitidos, pelo empregador, bem como receber capacitação na admissão e de forma contínua para garantir sua segurança ${ }^{30}$. É importante que a empresa cobre do colaborador o uso do EPI durante toda a jornada de trabalho, fornecendo adequação e conforto do equipamento, bem como o investimento em EPC, principalmente nas empresas de pequeno porte.

No entanto, vale ressaltar que o uso e disponibilidade de EPI e EPC não são os únicos fatores de prevenção de acidentes de trabalho. Os acidentes são também decorrentes de outras variáveis e processos, a exemplo: salários impróprios, instalações inadequadas, ausência de programas de promoção de saúde do trabalhador e prevenção de riscos, relações de trabalho, condições socioeconômicas e educação. Nesse sentido, a prevenção de acidentes de trabalho perpassa outros aspectos, como a promoção e a proteção da saúde do trabalhador por meio da vigilância de riscos nos ambientes e condições de trabalho, dos agravos à saúde e pela organização e prestação da assistência à saúde do trabalhador ${ }^{31,32}$.

Quanto ao horário da ocorrência de acidentes, neste estudo, verificou-se predominância nos períodos da manhã e da tarde. Em estudo realizado em um hospital de Teresina (PI), verificou-se que os acidentes de trabalho ocorreram no turno matutino (49\%), seguido do vespertino (41\%) e noturno (10\%), demonstrando também maior incidência no horário comercial $^{33}$. Entretanto, verificou-se que a variável de horário do acidente é ainda pouco explorada em estudos sobre o tema.

A evolução do caso, neste estudo, é marcada pela incapacidade temporária, achado também predominante em nível nacional $\mathbf{8 , 1 2 , 1 7 , 2 1}$. Considerando-se que a própria definição do acidente de trabalho grave já sugere que para sua classificação deve ocorrer repercussão funcional6, espera-se encontrar nas notificações o registro de incapacitação do profissional. Tal resultado sugere a maior parte dos acidentes estudados não chegam ao ponto de apresentar incapacidade permanente ou óbito. Tal resultado sugere que a maior parte dos acidentes estudados não chega ao ponto de apresentar incapacidade permanente ou óbito, mas também que pode não haver uma continuidade de acompanhamento dos casos para identificar se, a longo prazo, a lesão tida como temporária passou a ser permanente.

Quanto aos mecanismos do acidente e lesão, os CID mais frequentes foram aqueles relacionados às quedas e traumas, respectivamente, sendo a mão a parte do corpo mais atingida, assemelhando-se a outros estudos ${ }^{\mathbf{1 4}, \mathbf{3 4}}$.

Em um panorama nacional sobre acidentes de trabalho, os CID mais registrados e de maior 
frequência são aqueles associados a ferimentos ou fraturas em punho e mão, parte do corpo mais atingida em acidentes típicos ${ }^{8}$. No Brasil, a maior causa de afastamentos das atividades laborais por acidentes de trabalho é decorrente de indivíduos que tiveram o membro superior atingido, ou quase $50 \%$ dos casos ${ }^{12}$.

Quanto à mortalidade relacionada ao acidente de trabalho, não é esperado que o indivíduo seja exposto ao risco de morte em função da atividade laboral desempenhada. Nesse sentido, a legislação brasileira, por meio da CLT, normas regulamentadoras e legislação pertinente ao Sistema Único de Saúde, visa ao fortalecimento dos direitos, segurança e saúde do trabalhador ${ }^{30,35,36}$.

O achado de óbitos ( $n=39 / 2,2 \%$ ) relacionados ao acidente de trabalho corrobora resultados de outro estudo realizado em Pernambuco, que identificou uma taxa de $2,9 \%$ de mortalidade no estado ${ }^{16}$. Ainda corroborando o resultado ora encontrado, observou-se em uma série histórica que, no Brasil, para o período 1998 a 2008, foram registrados 27.429 óbitos por acidentes de trabalho, gerando uma taxa de mortalidade de 2,25\% ${ }^{27}$. Em estudo epidemiológico recente sobre a saúde do trabalhador em nível nacional, a mortalidade por acidente de trabalho ainda apresenta valores alarmantes em diferentes regiões brasileiras, incluindo o Paraná, tornando-se necessárias ações, estratégias e políticas em diferentes esferas que propiciem mudanças nesse cenário ${ }^{37}$.

Ainda, sobre a mortalidade por acidente de trabalho vale ressaltar a existência de subnotificações nos sistemas pertinentes. Um estudo que comparou os dados do Sinan com o Sistema de Informação sobre Mortalidade (SIM) em Belo Horizonte (MG) mostrou que existe a ocorrência de subnotificações de óbitos por acidentes de trabalho graves no Sinan ${ }^{38}$. A justificativa para tal constatação seria o fato de esses sistemas não se inter-relacionarem, sendo alimentados separadamente.

Segundo o Departamento de Informática do Sistema Único de Saúde do Brasil (Datasus) ${ }^{39}$ no município de Cascavel (PR), ocorreu, no período de 2012 a 2017, um total de 104 óbitos descritos nas declarações de óbitos como sendo motivados por acidente de trabalho, revelando uma diferença de pelo menos 65 óbitos entre os casos notificados nos dois sistemas, sugerindo que, no município de estudo, pode existir subnotificação.

No estado do Paraná, há um comitê de investigação de óbitos atuante, desenvolvido em 1997. Tem como fontes de dados as CAT notificadas, as Declarações de Óbito (DO) e o Sinan, cruzando dados dos sistemas de informação de todo o Estado ${ }^{40}$. No período de 1997 a 2007, foram registradas 1682 amputações, 1461 óbitos, 242 óbitos em trajeto, segundo o Comitê Estadual de Investigação de Óbitos e Amputações Relacionadas ao Trabalho no Paraná (Ceioart) ${ }^{\mathbf{4 1}}$.

No informativo mais recente do Comitê, no período de 1998 a 2011, houve 4952 acidentes de trabalho analisados, 2145 ocorrências de amputação por acidentes de trabalho, 1911 acidentes com óbito e 896 óbitos em acidente de trabalho em trânsito ${ }^{42}$.

Neste estudo, a ocupação mais frequente relacionada aos óbitos por acidente de trabalho grave foi a de pedreiro. A construção civil, em função das características das atividades desenvolvidas, é um ambiente de trabalho que oferece consideráveis riscos ao trabalhador, como a ocorrência de acidentes e óbito1,16,43. Corroborando a informação, em outro estudo, os autores afirmam que o trabalhador da construção civil está exposto aos acidentes graves de três a seis vezes mais, quando comparado a outros trabalhadores ${ }^{44}$.

Em um segundo momento deste estudo, na análise de agrupamento por similaridade, foi possível verificar que a maioria das variáveis quantitativas e qualitativas estudadas foram importantes na formação dos grupos, ou seja, que a maioria delas apresentou relação determinante e condicionante aos acidentes de trabalho, bem como com o desfecho. Excetuaram-se as seguintes variáveis: sexo, gestante, houve outros trabalhadores atingidos e se ocorreu atendimento médico. 
Os determinantes de saúde podem ser ambientais, econômicos, sociais, e estão relacionados ao estado de saúde da população ${ }^{45}$. Assim, é fundamental conhecer os fatores ou variáveis determinantes e condicionantes de saúde, i.e., moradia, educação, transporte, acesso dos bens e serviços, condições de trabalho, para a formulação de políticas públicas destinadas à promoção de saúde 35 .

Os profissionais de saúde devem ter embasamento sobre tais determinantes, pois podem atuar e agir de acordo com as necessidades da população, estabelecendo prioridades, bem como podem ser utilizados para acompanhar ou avaliar o processo da evolução dos agravos e das intervenções aplicadas no âmbito individual, institucional e social ${ }^{46}$.

O estudo do perfil epidemiológico de indivíduos que sofreram acidente de trabalho grave possibilitou identificar fatores que determinam a saúde e influenciam a ocorrência dos acidentes de trabalho. Tal resultado pode subsidiar a formulação de ações, estratégias e políticas públicas direcionadas a esse grupo específico.

Em relação à identificação das variáveis ligadas estatisticamente ao óbito, o maior risco foi atribuído ao acometimento do membro superior no acidente e à classificação por traumatismos de múltiplas lesões. Embora esse seja o resultado encontrado neste estudo, tal achado diverge de outros encontrados na literatura, que, apesar de relacionarem traumatismo ao óbito, informam que a região mais atingida comumente e mais relacionada à fatalidade em acidentes de trabalho grave são a cabeça, tórax e membros inferiores ${ }^{\mathbf{1 5}, 19}$.

Quanto à variável ligada ao menor risco de óbito, o preenchimento da CAT marcada como ignorada pode ser decorrente do preenchimento inadequado da ficha de notificação compulsória, bem como da questão conceitual sobre a definição de acidente de trabalho entre a previdência social e o Sinan, gerando diferença entre as notificações nos dois sistemas ${ }^{5,9}$.

Achados desta pesquisa podem ter sido condicionados por essas incompletudes das fichas, sendo dois deles relevantes para compor o perfil neste estudo, a saber: i) os óbitos estão estatisticamente relacionados ao acometimento do membro superior; ii) acidentes graves acontecerem predominantemente em homens, empregados domésticos nos serviços gerais. Ambos os resultados não são corroborados pela literatura produzida em nível nacional, merecendo, assim, novos estudos envolvendo inclusive outras fontes de dados para fins de comparação, corroboração ou cruzamento estatístico como exemplo CAT notificado e DO.

O preenchimento adequado dessas fichas oferece suporte para alimentação do Sinan de maneira mais fidedigna e, consequentemente, informações mais robustas na geração de indicadores que subsidiarão conhecimento de base territorial para a tomada de decisões e planejamento em saúde. No estudo, durante o levantamento dos dados verificou-se a existência de campos não preenchidos ou marcados como ignorados. Nesse sentido, a equipe de saúde tem papel fundamental no preenchimento das fichas de notificações de doenças e agravos de notificação compulsória ${ }^{4}$, sendo importante a conscientização dos profissionais e o treinamento adequado.

\section{Conclusões}

Este estudo revela que os resultados obtidos acerca do perfil de notificações de acidente de trabalho grave no município de Cascavel (PR), convergem com as estatísticas disponíveis em nível estadual e nacional e com resultados de outros estudos científicos realizados em diversas regiões do Brasil. Ressalta-se que, como limitação, houve a subnotificação do agravo, sobretudo do Sinan em relação ao SIM.

O conhecimento do perfil epidemiológico desses trabalhadores acidentados mostrou a predominância dos acidentes em uma faixa etária produtiva, sexo masculino, brancos, ensino fundamental incompleto, residentes na área urbana de Cascavel (PR). Esses trabalhadores exerciam, principalmente, a ocupação de empregado doméstico de serviços gerais e, em 
sua maior parte, sofreram acidentes típicos nas instalações do contratante. A maioria possuía carteira de trabalho assinada, embora, em mais da metade dos casos, a CAT não foi preenchida. Os acidentes ocorreram em sua maioria em trabalhadores com tempo na função inferior a um ano e em horário comercial.

A maior parte dos acidentados recebeu atendimento médico, e o maior número de notificações ocorreu na unidade hospitalar. Destaca-se a mão como a parte do corpo mais atingida, o regime de tratamento hospitalar e a evolução dos casos para a incapacidade temporária. Ocorreram 39 óbitos entre esses trabalhadores notificados para acidente de trabalho grave. A maioria das variáveis estudadas foram significativas para ocorrência dos acidentes e desfechos dos casos, incluindo os óbitos.

O conhecimento do perfil epidemiológico de uma dada população, bem como dos fatores determinantes de saúde, contribui para subsidiar o planejamento em saúde nas localidades estudadas e para melhor compreensão do comportamento do agravo. Para realização de pesquisas neste enfoque, podem-se utilizar sistemas de informação em saúde disponíveis no Brasil. Neste estudo, utilizou-se o Sinan.

Durante o estudo, observou-se o não preenchimento ou forma inadequada de preenchimento de alguns campos das fichas de notificação dos acidentes. Elas alimentam o sistema de informação, que fornecerá subsídios acerca do agravo e do indivíduo que foram notificados. Também foi verificada a divergência entre os sistemas de informações.

Nesse sentido, ressalta-se a importância do preenchimento adequado das notificações, o que permitirá a correta alimentação do sistema de informação e a composição de dados mais fidedignos, que, por sua vez, subsidiaram a elaboração de ações, estratégias e políticas de prevenção, promoção e assistência à saúde do trabalhador.

Para finalizar, é necessária a capacitação permanente dos profissionais de saúde, em todos os níveis de atenção à saúde, acerca do conhecimento do perfil epidemiológico pertinente ao agravo em âmbito local, estadual e nacional; bem como suas especificidades como agravo de notificação compulsória, e de ações em Visat; além de novos estudos acerca do tema, em função da sua importância.

\section{Agradecimentos}

Agradecimento à Universidade Estadual do Oeste do Paraná e ao setor de Vigilância em Saúde do Trabalhador do Município de Cascavel (PR).

\section{Colaboradoras}

Zack BT (0000-0003-1020-0535)* contribuiu substancialmente para a concepção e o planejamento e para a análise e a interpretação dos dados. Ross C (0000-0003-0540-1455)* contribuiu significativamente para a elaboração da revisão crítica do conteúdo quanto orientador. Gouvêa LAVN (0000-0001-66417114)* e Tonini NS (0000-0003-4704-7634)* contribuíram para a aprovação da versão final do manuscrito mediante papel de banca do Trabalho de Conclusão de Residência da primeira autora. 


\section{Referências}

1. Cavalcante CAA. Cossi MS. Oliveira Costa, et al. Análise crítica dos acidentes de trabalho no Brasil. RAS [internet]. 2015 [acesso em 2019 mar 3]; 13(44):100109. Disponível em: http://seer.uscs.edu.br/index. php/revista_ciencias_saude/article/view/2681.

2. Brasil. Ministério da Saúde. Portaria n ${ }^{\circ} 777$, de 23 de abril de 2014. Dispõe sobre os procedimentos técnicos para a notificação compulsória de agravos à saúde do trabalhador em rede de serviços sentinela específica, no Sistema Único de Saúde - SUS. Diário Oficial da União. 24 Abr 2004.

3. Brasil. Ministério da Saúde. Portaria ${ }^{\circ} 1823$, de 23 de agosto de 2012. Institui a Política Nacional de Saúde do Trabalhador e da Trabalhadora. Diário Oficial da União. 23 Ago 2012.

4. Brasil. Ministério da Saúde. Portaria nº 264, de 17 de fevereiro de 2020. Altera a Portaria de Consolidação $n^{\circ} 4 / G M / M S$, de 28 de setembro de 2017, para incluir a doença de Chagas crônica, na Lista Nacional de Notificação Compulsória de doenças, agravos e eventos de saúde pública nos serviços de saúde públicos e privados em todo o território nacional. Diário Oficial da União. 17 Fev 2016.

5. Brasil. Lei $\mathrm{n}^{\circ}$ 8.213, de 24 de julho de 1991. Dispõe sobre os Planos de Benefícios da Previdência Social e dá outras providências. Diário Oficial da União. 24 Jul 1991.

6. Brasil. Ministério da Saúde. Secretaria de Atenção à Saúde. Notificação de acidentes do trabalho fatais, graves e com crianças e adolescentes [internet]. Brasília, DF: Ministério da Saúde; 2006. [acesso em 2019 dez 21]. Disponível em: http://bvsms.saude.gov.br/ bvs/publicacoes/06_0442_M.pdf.

7. Brasil. Ministério da Saúde. Sistema de Informação de Agravos de Notificação - SINAN [internet]. [acesso em 2019 out 20]. Disponível em: http://portalsinan.saude.gov.br/o-sinan.
8. Brasil. Ministério da Previdência Social. Anuário Estatístico da Previdência Social, 2017 [internet]. 2017. [acesso em 2019 dez 20]. Disponível em: http://www. previdencia.gov.br/2018/11/secretaria-lanca-anuario-estatistico-da-previdencia-social-2017/.

9. Paraná. Secretaria de estado de saúde. Boletim Epidemiológico da saúde do trabalhador no Paraná: notificações dos agravos da saúde do trabalhador no Paraná (2006 a 2016) [internet]. 2017 nov [acesso em 2019 dez 21]. Disponível em: http://www.saude.pr.gov.br/ arquivos/File/BOLETIM.pdf.

10. Organização Mundial da Saúde. Classificação Estatística Internacional de Doenças e Problemas Relacionados à Saúde (CID-10). 10 rev. São Paulo: Universidade de São Paulo; 2009.

11. Organização Internacional do Trabalho. OIT Notícias: Brasil [internet]. 2016. [acesso em 2019 out 20]. Disponível em: http://www.ilo.org/brasilia/lang-pt/ index.htm.

12. Possebom G, Alonço AS. Panorama dos acidentes de trabalho no Brasil. Nucleus [internet]. 2018 [acesso em 2020 jan 30]; 15(2):15-22. Disponível em: http:// nucleus.feituverava.com.br/index.php/nucleus/article/view/2691.

13. Brasil. Ministério Público do Trabalho. Observatório de Segurança e Saúde no Trabalho [internet]. 2020 [acesso em 2020 jul 11]. Disponível em: https://smartlabbr.org/sst/localidade/4104808.

14. Miranda FMDA, Scussiato LA, Kirchhof ALC. Caracterização das vítimas e dos acidentes de trabalho fatais. Revista Gaúcha de Enfermagem [internet]. 2012 [acesso em 2019 nov 10]; 33(2):45-51. Disponível em: https://www.seer.ufrgs.br/RevistaGauchadeEnfermagem/article/view/22026.

15. Malta DC, Stopa SR, Silva MMAD, et al. Acidentes de trabalho autorreferidos pela população adulta bra- 
sileira, segundo dados da Pesquisa Nacional de Saúde, 2013. Ciênc. Saúde Colet. [internet]. 2017 [acesso em 2019 mar 3]; (22):169-178. Disponível em: http:// dx.doi.org/10.1590/1413-81232017221.17862015.

16. Campos AG, Gurgel ADM. Acidentes de trabalho graves e atividades produtivas nas regiões administrativas de saúde em Pernambuco: uma análise a partir da identificação de aglomerados produtivos locais. Rev. Bras. Saúde Ocupac. [internet]; 2016 [acesso 2020 jan 15]:(41). Disponível em: link http://www.scielo.br/scielo.php?pid=S0303$-76572016000100208 \&$ script=sci_arttext\&tlng=pt.

17. Cardoso MG, Romero LO, Bachi ZC, et al. Caracterização das ocorrências de acidentes de trabalho graves. Arquiv. Ciênc. Saúde [internet]. 2016 [acesso em 2019 set 6]; 3(4):83-88. Disponível em: http://www. cienciasdasaude.famerp.br/index.php/racs/article/ view/502.

18. Silva AR, Araújo TMD. Acidentes de trabalho grave no estado da Bahia no período de 2007 a 2012. Rev. Baiana de Saúde Pública [internet]. 2017 [acesso em 2020 jan 2]; (2):57-69. Disponível em: http://rbsp.sesab.ba.gov.br/index.php/rbsp/article/view/2694.

19. Scussiato LA, Sarquis LMM, Kirchhof ALC, et al. Perfil epidemiológico dos acidentes de trabalho graves no Estado do Paraná, Brasil, 2007 a 2010. Epidemiologia e Serviços de Saúde [internet]. 2013 [acesso em 2019 mar 2]; 22(4):621-630. Disponível em: http:// scielo.iec.gov.br/scielo.php?pid=S16794974201300 0400008\&script=sci_arttext\&tlng=en.

20. Instituto Brasileiro de Geografia e Estatística. Apresentação PNAD contínua - Educação. Estatísticas Sociais. [internet]. 2017 [acesso em 2020 jan 20]. Disponível em: https://agenciadenoticias.ibge.gov.br/ agencia-detalhe-de-midia.html?view=mediaibge \& catid $=2103 \& \mathrm{id}=1657$.

21. Marinho CDS. Consequências econômicas em saúde com acidentes de trabalho: realidade do município de Piracicaba [tese]. São Paulo: Universidade de São Paulo; 2017. 147 p.
22. Brasil. Ministério do Trabalho. Classificação Brasileira de Ocupações. [internet]. 2020. [acesso em 2020 jul 11]. Disponível em: http://www.mtecbo.gov.br/cbosite/pages/pesquisas/BuscaPorTituloResultado.jsf.

23. Fontana LO, Grillo LP. Perfil dos acidentes de trabalho em uma cidade de pequeno porte no sul do Brasil. Saúde (Santa Maria). [internet]. 2018 [acesso em 2020 jan 28]; 44(1). Disponível em: https://periodicos.ufsm.br/revistasaude/article/view/23894/pdf.

24. Paraná. Instituto Paranaense de Desenvolvimento Econômico e Social - IPARDES. Saldo do emprego com carteira assinada no Paraná - janeiro 2001 - novembro 2019. [internet]. 2019 [acesso em 2020 jan 5]. Disponível em: http://www.ipardes.pr.gov.br/sites/ ipardes/arquivos_restritos/files/documento/2019-12/ emprego_formal.pdf.

25. Júnior R. Acidentes de trabalho graves envolvendo membros superiores notificados em um Centro de Referência em Saúde do Trabalhador [dissertação] [internet]. Uberlândia: Universidade Federal de Uberlândia; 2018. [acesso em 2020 jan 30]. Disponível em: http://dx.doi.org/10.14393/ufu.di.2018.990.

26. Pialarissi R. Precarização do trabalho. Revista de Administração em Saúde [internet]. 2017 [acesso em 2020 jan 28]; 17(66). Disponível em: http://cqh.org. br/ojs-2.4.8/index.php/ras/article/view/11.

27. Almeida FS, Morrone LC, Ribeiro KB. Tendências na incidência e mortalidade por acidentes de trabalho no Brasil, 1998 a 2008. Cad. Saúde Pública [internet]. 2014 [acesso em 2019 mar 2]; (30):1957-1964. Disponível em: http://www.scielo.br/scielo.php?pid=S010211X2014000901957\&script=sci_abstract\&tlng=pt.

28. Instituto Brasileiro de Geografia e Estatística. Características Adicionais do Mercado de Trabalho 2018 [internet]. 2018. [acesso em 2020 jan 25]. Disponível em: https://biblioteca.ibge.gov.br/visualizacao/ livros/liv101694_informativo.pdf.

29. Meireles NM, Oliveira Pinto F. A conscientização do trabalhador quanto à importância do uso do EPI na aerosoldas em Macaé. Interdiscip. Scient. J. [internet]. 
2016 [acesso em 2019 nov 2]; 3(1):46-62. Disponível em: http://revista.srvroot.com/linkscienceplace/index.php/linkscienceplace/article/viewFile/221/133.

30. Brasil. Ministério do Trabalho e Emprego. Norma Regulamentadora $n^{\circ} 32$. Segurança e saúde no trabalho em serviços de saúde. Diário Oficial da União. 15 Nov 2005.

31. Lara R. Saúde do trabalhador: considerações a partir da crítica da economia política. Revista Katálysis [internet]. 2011 [acesso em 2020 ago 24]. 14(1):7885. Disponível em: https://doi.org/10.1590/S141449802011000100009.

32. Rouquayrol MZ. Rouquayrol: epidemiologia \& saúde. 8. ed. Rio de Janeiro: Meedbook; 2018.

33. Ribeiro PC, Ribeiro ACC, Lima Júnior FPB. Perfil dos acidentes de trabalho em um hospital de Teresina, PI. Cogitare Enfer. [internet]. 2010 [acesso em 2020 jan 25]; 15(1). Disponível em: https://revistas. ufpr.br/cogitare/article/view/17181.

34. Galdino A, Santana VS, Ferrite S. Qualidade do registro de dados sobre acidentes de trabalho fatais no Brasil. Rev. Saúde Púb. [internet]. 2017 [acesso em 2020 jan 25]; 51(120). Disponível em: https://www. scielosp.org/article/rsp/2017.v51/120/pt/.

35. Brasil. Lei $n^{\circ} 8.080$, de 19 de setembro de 1990.Dispõe sobre as condições para a promoção, proteção e recuperação da saúde, a organização e o funcionamento dos serviços correspondentes e dá outras providências. Diário Oficial da União. 19 Set 1990.

36 Brasil. Decreto-lei no 5.452, de 1 de maio de 1943. Aprova a Consolidação das Leis do Trabalho. Diário Oficial da União. 1 maio 1943.

37. Brasil. Ministério da Saúde. A epidemiologia da saúde do trabalhador no Brasil [internet]. Salvador: Universidade Federal da Bahia; 2020. [acesso em 2020 ago 18]. Disponível em: https://www.saude.gov.br/ images/pdf/2020/April/27/epidemiologia-saude-trabalhador-ufba-ms-4mar20-isbn.pdf.
38. Drumond EDF, Silva JDM. Avaliação de estratégia para identificação e mensuração dos acidentes de trabalho fatais. Ciênc. Saúde Colet. [internet]. 2013 [acesso em 2020 jan 15]; 18:1361-1365. Disponível em: https://www.scielosp.org/article/csc/2013. v18n5/1361-1365/pt/.

39. Brasil. Ministério da Saúde. Departamento de informática do SUS (DATASUS). Óbitos por causas externas - Paraná. [internet]; 2019. [acesso em 2020 jan 20]. Disponível em: tabnet.datasus.gov.br/CGI/tabcgi.exe?sim/cnv/ext10pr.def.

40. Ramos Junior AJ. Comitê de Investigação de Óbitos e Amputações Relacionados ao Trabalho do Estado do Paraná: uma experiência coletiva de produção de conhecimento e estruturação de política de saúde do trabalhador. [dissertação] [internet]. Curitiba: Universidade Federal do Paraná; 2001. [acesso em 2020 jul 11]. Disponível em: https://acervodigital.ufpr.br/ bitstream/handle/1884/11303/?sequence=1.

41. Paraná. Comitê Estadual de Investigação de Óbitos e Amputações Relacionadas ao Trabalho no Paraná (Ceioart). [internet]. 2020. [acesso em 2020 jul 12]. Disponível em: https://fdocumentos.tips/document/ comite-estadual-de-investigacao-de-obitos-e-amputacoes-relacionados-ao-trabalho-do-parana-ceioart. html.

42. Paraná. Secretaria de Estado da Saúde do Paraná. Superintendência de Vigilância em Saúde. Caderno temático de vigilância de violências e acidentes no Paraná. Curitiba: SESA/SVS. [internet]. 2014 [acesso em 2020 jul 12]. Disponível em: http://www.saude.pr.gov.br/busca?termo=ceioart.

43. Mendes PC, Magalhães SF. Perfil do trabalhador vítima de acidente grave na construção civil de 2013 a 2015 em Uberlândia-MG. In: Anais IX Simpósio Nacional de Geografia da Saúde - Geosaude; 2019 Jul 19-21; Minas Gerais. Minas Gerais: Instituto Federal Catarinense; 2019. [acesso em 2020 jul 12]. Disponível em: http://inscricao.eventos.ifc.edu.br/index. php/geosaude/geosaude/paper/view/1418. 
44. Gürcanli GE. Müngen U. Analysis of construction accidents in Turkey and responsible parties. Industrial health [internet]. 2013 [acesso em 2020 jan 12]; 51(6):581-595. Disponível em: http://www.ncbi.nlm. nih.gov/pmc/articles/PMC4202747.

45. Carrapato P, Correia P, Garcia B. Determinante da saúde no Brasil: a procura da equidade na saúde. Saúde e Sociedade [internet]. 2017 [acesso em 2019 dez 15]; (26):676-689. Disponível em: https://doi.org/10.1590/ S0104-12902017170304.
46. Henriques A, Alves P, Gomes, et al., V. O enfermeiro do trabalho na análise de determinantes de saúde dos trabalhadores. Rev. Portug. de Saúde Ocup. [internet]. 2016 [acesso em 2020 jan 30]; (1):39-51. Disponível em: https://10.31252/RPSO.22.02.2016.

Recebido em 01/03/2020

Aprovado em 28/08/2020

Conflito de interesses: inexistente

Suporte financeiro: não houve 